\title{
Educación Física, Calidad de Vida y la Nueva Sociología de la Infancia: Repensando la Metodología Mixta en Sociología \\ Physical Education, Quality of Life and New Sociology of Childhood: Rethinking about Mixed Methodology in Sociology \\ Fidel Molina \\ Universidad de Lleida (España)
}

Resumen: La Educación física tiene una relación directa con cuatro de las nueve dimensiones que componen la Calidad de Vida (Eurostat, 2013): salud, educación, ocio y relaciones sociales y bienestar subjetivo. La Educación física aparece como un elemento fundamental para indicadores importantes en la medición de la calidad de vida, de presente y de futuro. Y es que la Educación física puede proporcionar una socialización adecuada en hábitos saludables, patrones de conducta relacionales y mejor autopercepción del bienestar a niños y jóvenes que no son «objetos» de estudio, sino sujetos activos y participantes, como nos plantea la nueva sociología de la infancia. La nueva sociología de la infancia es una subdisciplina de la sociología (de la educación física y del deporte) que estudia la realidad holística de la vida de los niños y jóvenes. En este sentido, también analiza elementos fundamentales de su calidad de vida presente, en relación con ellos mismos. La metodología mixta es una opción global y completa que se presenta como opción muy adecuada en diversos tipos de investigaciones que aúnan salud, educación y aspectos sociales y culturales. Las conclusiones muestran cómo desde la Educación Física y mediante la metodología mixta (encuestas, entrevistas, grupos de discusión, etnografía e investigación-acción), se puede analizar de manera más completa algunos de los indicadores de calidad de vida entre niños y jóvenes, en relación con ellos mismos y en planteamientos intergeneracionales, de presente (de los propios niños y jóvenes «aquí y ahora») y de futuro (cuestiones estructurales de base socioeducativa).

Palabras clave: Sociología de la Educación, Educación Física, Nueva Sociología de la Infancia, Calidad de Vida, Metodología Mixta, InvestigaciónAcción.

Abstract: Physical Education has a direct relationship with four of the nine dimensions that constitute Quality of life (Eurostat, 2013): health, education, leisure and social relationships, and personal welfare. The New Sociology of Childhood is a sub-discipline of Sociology (of Physical Education) that explores children's and young people's life in a holistically manner. Physical Education appears as a fundamental element for measuring quality of life. Thus, Physical Education can provide children and youth with positive socialization into healthy habits, relational patterns and better self-perception of welfare: they are not 'objects' of research, but they are active «subjects». In this sense, this sub-discipline also analyses key elements of quality of life as well as it studies their relation with each other. Mixed methodology is a suitable methodology in different types of research about health, education and socio-cultural aspects. The outcomes show how Physical Education (through Mixed methodology: surveys, interviews, discussion groups, ethnography and action-research) can analyse children's and youth's indicators of quality of life, both in relation with each other and in intergenerational approaches, looking at the Present (children and young, «here and now») and at the Future (structural issues from socio-educational basis).

Key words: Sociology of Education, Physical Education, New Sociology of Childhood, Quality of Life, Mixed Methods, Action Research.

\section{Introducción: Calidad de vida y Educación Física}

La calidad de vida es un concepto multidimensional que se mide a través de una serie de indicadores. Entre estos indicadores, en mayor o menor medida, se encuentran unos ámbitos relacionados con la Educación Física.

En este sentido, Eurostat publicó en 2013 un primer conjunto de Indicadores de Calidad de Vida(Quality of Life) que el Instituto Nacional de Estadística (INE) ha acomodado para el caso español, analizando las diferentes dimensiones que componen la calidad de vida. Estas nueve dimensiones son las siguientes: condiciones materiales de vida, trabajo, salud, educación, ocio y relaciones sociales, seguridad física y personal, gobernanza y derechos básicos, entorno y medioambiente y bienestar subjetivo.

La Educación física y el deporte tienen una relación directa con cuatro de estas dimensiones: salud, educación, ocio y relaciones sociales y bienestar subjetivo. La Educación física aparece como un elemento fundamental para indicadores importantes en la medición de la calidad de vida, de presente y de futuro.

Por otra parte, la Sociología enmarca una visión holística de las interrelaciones que se dan entrela Educación, la Salud, la Calidad de Vida y, en nuestro caso, con la infancia, la adolescencia y la juventud. Los hábitos saludables socializados en la infancia son buenos predictores de futuro en cuanto a la calidad de vida de la población. Para ello no sólo debemos investigar «sobre» la infancia, la adolescencia y la juventud, sino también investigar «con» ellos (a lo largo de todo el documento utilizamos el masculino genérico para referirnos igualmente al género

Fecha recepción: 25-07-16. Fecha de aceptación: 25-04-17 Fidel Molina

molina@geosoc.udl.cat masculino y femenino), los niños, adolescentes y jóvenes. No son únicamente objetos de estudio sino, sobre todo, sujetos en acción.

En esta línea, la orientación metodológica propuesta ante investigaciones de este tipo es la denominada metodología mixta, esto es, una metodología que imbrica lo cuantitativo con lo cualitativo. Es un enfoque completo y complejo, que no es únicamente la suma de unas orientaciones cuantitativas por un lado y otras cualitativas por otro, sino que el enfoque inicial ya prevé dicha complementariedad mutuamente enriquecedora y necesaria.

En todo caso, se trata de analizar de manera completa (interconectada) algunos de los indicadores de calidad de vida entre niños y jóvenes, en relación con ellos mismos y en planteamientos intergeneracionales, de presente (de los propios niños y jóvenes «aquí y ahora») y de futuro (cuestiones estructurales de base socioeducativa), que puedan coadyuvar a propuestas y políticas educativas que socialicen en hábitos saludables, y en el incremento y el gusto por la actividad física.

\section{La Sociología de la Educación Física y la Nueva Sociología de la Infancia}

La Educación física puede proporcionar una socialización adecuada en hábitos saludables, patrones de conducta relacionales y mejor autopercepción del bienestar a niños y jóvenes que no son «objetos» de estudio, sino sujetos activos y participantes, como nos plantea la nueva sociología de la infancia.

La «nueva sociología de la infancia» es una subdisciplina de la sociología (de la educación física y del deporte), emparentada, de alguna manera, con la «nueva sociología de la educación», y que estudia la realidad holística de la vida de los niños y jóvenes. En este sentido, también analiza elementos fundamentales de su calidad de vida presente, en relación con ellos mismos. 
A lo largo de los años 70 y los 80 del siglo pasado, la denominada «nueva sociología de la educación» planteaba una posición crítica anteel funcionalismo y su macrosociología, por entender que es muy determinista en su análisis de la acción social (y del ser humano), apareciendo el sistema educativo sólo como un agente de transmisión de valores y de selección de alumnos. En cambio, los «nuevos sociólogos» destacan la concepción del ser humano como un ser determinado y determinante. En este sentido, también debemos mencionar la corriente interaccionista norteamericana que enfatiza el carácter interactivo e intersubjetivo de la vida social.

La «nueva sociología de la educación» ha sido identificada frecuentemente con la sociología del conocimiento, y a través del análisis microsociológico se interesa por la interpretación que los actores sociales realizan de su acción en la actividad cotidiana, como agentes activos que construyen la realidad en que se insieren (Berger y Luckmann, 1978). Por otro lado, a parte de los factores sociales extraescolares, también se considera como un elemento fundamental la interacción de los actores del sistema escolar. A través de dicha interacción los actores participan en la construcción, selección y gestión de los conocimientos que, así, pueden cambiarse en la escuela. Aquí radica una diferencia fundamental, con relación a la «vieja» sociología de la educación: ahora el papel de los enseñantes, por ejemplo, adquiere una relevancia fundamental en los cambios educativos; pueden convertirse en investigadores, en lugar de ser meros transmisores de conocimientos que le vienen dados; y, en esta línea, a los alumnos (niños y jóvenes) con un papel activo, como sujetos de la educación (socialización).

En el entramado entre Sociología, Educación y Actividad Física, vislumbramos la posibilidad de integrar dentro del ya clásico ámbito de la Sociología de la Educación, un apartado de Sociología de la Educación Física, muy ligada a aspectos culturales y próxima a la cada vez más consolidada idea de la calidad de vida. Todo ello lo plantemos así porque elámbito de la calidad de vida en lo humano es sociológico, tiene mucho que ver con la socialización (educación) y no podemos obviarlo entre enfoques hiperracionalistas o irracionalistas (vid Molina, 2007, 2012).

Los procesos de socialización vienen definidos por la tensión entre la interiorización normativa y cultural, y el distanciamiento crítico(Dubet y Martuccelli, 1996: 511), o dicho de otro modo, entre el influjo de las instituciones sociales sobre los individuos y la modificación de aquellas por la acción de éstos. El análisis de la socialización como estudio de los procesos a través de los que las estructuras sociales influyen sobre las conductas individuales, pero también de las transformaciones de la sociedad gracias a la acción deliberada o no de los individuos, tiene varias interpretaciones según diversos paradigmas sociológicos. Durkheim señala que la socialización perpetúa y refuerza la necesaria homogeneidad cultural entre los miembros de una determinada sociedad. Simmel, por otra parte, define la sociedad como el complejo de individuos socializados, conformados socialmente, pero también como suma de las formas de relación mediante las cuales surge ella misma, es decir, que somos productos de la sociedad pero también miembros de ella. Bernstein, por ejemplo, define la socialización como un mecanismo más o menos arbitrario, dirigido a transformar lo que es cultural (lo que se ha construido socialmente) en natural. Otros autores, retomando la idea de la tensión entre la interiorización normativa y cultural y el distanciamiento crítico, agrupan las diversas teorías de la socialización en dos grandes bloques que siguen estos dos postulados básicos. Así el primero, más cercano a la modernidad, es el que afirma la reversibilidad de la subjetividad de los actores y la objetividad del sistema, recalcando que la socialización se define como interiorización normativa y cultural; el segundo, más cercano a los postulados de la postmodernidad, aparece como más sensible a la heterogeneidad cultural y social, privilegiando el tema del distanciamiento, de la actividad de los individuos, de la separación entre el actor y el sistema (Dubet y Martuccelli, 1996: 511 y s.). En definitiva nos movemos entre situaciones que imbrican al individuo y a la sociedad. En este sentido, podemos reconocer en los procesos de socialización las posibilidades individuales y sociales de la calidad de vida (y sus ámbitos y/o indicadores).

En este sentido, los procesos de socialización tienen lugar a lo largo de toda la vida y significan la integración social en una comunidad con una serie de valores, normas, actitudes, pautas de comportamiento, conocimientos, etc... El modelo tradicional ha sido el que reconocía que básicamente, la socialización se realizaba desde los adultos a los más jóvenes. Es el modelo que explicaba Durkheim como definición de la educación.

Sin embargo, Margaret Mead indica que aunque este modelo es preponderante y fundamental, sólo representa uno de los tres que se suelen dar, según la cultura imperante. Así, este modelo tradicional se da en sociedades de cultura «postfigurativa», donde la importancia en los procesos de socialización la tiene la experiencia vital, los años y las experiencias vividas. No obstante, también tenemos el modelo de las sociedades de cultura «cofigurativa» en que lo importante para transmitir conocimientos, pautas de comportamiento, normas, etc... no es la experiencia vital de los adultos, sino la experiencia del «experto», del que está preparado en cada aspecto concreto; es una socialización más horizontal. Por último, en las sociedades de cultura «prefigurativa» son los jóvenes los que marcan las pautas educativas, transmitiendo una serie de valores, conocimientos y pautas de comportamiento a los adultos. El elemento común es la plasticidad y la flexibilidad que tienen los jóvenes para adaptarse y para integrar cuestiones innovadoras y de cambio vertiginoso, como pueden ser las TIC con sus constantes cambios en la informática, en el sonido y la imagen, etc... o planteamientos de hábitos y estilos de vida (saludables), por ejemplo.

En este sentido, la socialización prefigurativa, desde la juventud, implica cómo el gusto por el deporte (y la participación activa) pueden provocar cambios en las familias, y puede ayudar a la integración social (no exenta de tensiones y contradicciones). Podemos presentar como paradigmático el guión de la película «Quiero ser como Beckham», que relata las dificultades, pero también las posibilidades de integración social intercultural, de una chica británica de origen hindú que quiere jugar (y juega) al fútbol. La decisión de la joven provoca un debate en el seno de la familia (y de la comunidad) muy enriquecedor.

El tiempo de ocio debe ser un tiempo formativo, de integración social, de aumento de la calidad de vida y la salud. En este sentido, las administraciones locales, autonómicas y estatales (en el ámbito español y europeo, por ejemplo) suelen tener ejemplos de «buenas prácticas» al facilitar la práctica deportiva entre todos los ciudadanos (Molina, 2004, 2010).

En definitiva, se da una sociología de la infancia porque esta es un fenómeno social (Gaitán, 2006; Rodríguez, 2007; Salas, 2010). De hecho, hay autores como Corsaro que destacan que los niños no se limitan a adaptarse pasivamente, sino que participan activamente en las rutinas cotidianas que se les ofrecen en su entorno social, luego se apropian y reinterpretan sus elementos y, así, a través de una «reproducción interpretativa», contribuyen a la reproducción cultural y al cambio (Gaitán, 2006: 14). La acción formativa y educativa de la práctica deportiva suele tener un momento privilegiado que es la escolarización: es fundamental realizar los esfuerzos necesarios para que pueda ser una realidad el deporte para todos. Hay anécdotas contadas por padres de alumnos que pueden trascender a categoría de paradigma y ejemplo ilustrativo, sobre cómo la práctica deportiva de los hijos acaba promoviendo dicha práctica entre los padres o la familia, en general. Desde los que con el balón de fútbol o baloncesto «inician» (o «recuperan») a los padres en dichos juegos, a los que se han comprado una bicicleta para «entrenar» junto con sus hijos, porque éstos realizaban una salida escolar («travesía») de 5 días por el Delta del Ebro en bicicleta. Ejemplos bastante cotidianos, y más comunes y frecuentes de lo que parece, de una socialización prefigurativa en la práctica deportiva y la mejora de la calidad de vida y la salud.

En este sentido, hay estudios que destacan la importancia que se le concede a la práctica regular de actividad física sobre el autoconcepto físico y la autoeficacia social, mejorando, en términos generales, los procesos de desarrollo de los niños y niñas (Kyle, Hernández Mendo, Reigal Garrido, Morales Sánchez, 2016). Incluso, la propia valoración de la escuela tiene relación con la práctica de la actividad física de los escolares (Fernández Villarino, González Valero, Toja y Corrimode, 
2017). Además, parece indudable que el ámbito familiar inactivo constituye factor de riesgo en la actividad físico-deportiva de los adolescentes (Piéron y Ruiz-Juan, 2013). A partir de ahí, también tendrá lugar un reforzamiento sinérgico desde los jóvenes a los adultos y, en relación con la calidad de vida sobre todo en lo que respecta a hábitos y estilos de vida saludables, la influencia de la Educación Física en los niños y jóvenes y de éstos en su entorno más inmediato (familia, comunidad, etc.) puede ser crucial.

\section{Orientaciones desde la metodología mixta para la investiga- ción en Educación Física y Calidad de Vida}

La metodología mixta es una opción global y completa que se presenta como opción muy adecuada en diversos tipos de investigaciones que aúnan salud, educación y aspectos sociales y culturales. Es un planteamiento que comporta aspectos metodológicos cuantitativos y cualitativos, de forma integrada. Se trata de integrar tanto los aspectos cuantitativos como cualitativos, articulándose de manera complementaria y necesaria.

La metodología mixta es una opción holística, global y completa que se plantea como opción muy adecuada en diversos tipos de investigaciones que aúnan educación, salud y aspectos sociales y culturales. Es un planteamiento que comporta aspectos metodológicos cuantitativos y cualitativos, de forma integrada.

Es una idea de complementariedad entre las diversas técnicas cuantitativas y cualitativas, pero es una opción metodológica de primer orden. No es una mera yuxtaposición de técnicas cuantitativas por un lado y de técnicas cualitativas por otro, como en dos apartados diferenciados. Al contrario, es una opción metodológica de proyecto de investigación.

Se trata de integrar tanto los aspectos cuantitativos como cualitativos. En este sentido, desde un inicio y, teniendo en cuenta las preguntas de investigación y los objetivos que nos planteamos, vemos que la opción metodológica es mixta, conjugando desde el principio determinadas técnicas cualitativas y cuantitativas que se articulan de manera complementaria y necesaria.

La investigación acción es la base fundamental para la imbricación entre Educación Física y Calidad de Vida, y en la investigación para la mejora de todo ello. Aún cuando esta opción metodológica suele aparecer como cualitativa, puede incluir todos los componentes cuantitativos necesarios (encuestas, estadísticas, bases de datos), integrados en dicho planteamiento holístico e integrador.

En este sentido, la investigación acción es en sí misma una técnica de investigación que puede incluir otras técnicas, básicamente cualitativas (entrevistas en profundidad, grupos de discusión, la propia observación participante, etc.), pero también cuantitativas (cuestionarios, etc.) como acabamos de mencionar.

Lo fundamental de la investigación-acción es que se trata de una investigación para mejorar lo investigado, en la que los propios investigadores están, de una manera más o menos directa, implicados en dicha realidad. Las cuatro fases fundamentales son: la observación, el análisis, las propuestas de mejora, la acción. La evaluación se da a lo largo de todo el proceso y también se da una evaluación propiamente de resultados. Pero es un proceso, si se quiere, casi «sin fin», puesto que de la propia evaluación pueden surgir nuevos ciclos de análisis, propuestas y acciones de mejora.

Pueden haber diferentes ejemplos de investigación-acción en el campo de la educación, la salud y la calidad de vida: desde acciones en comunidades para empoderar a la población en relación con hábitos saludables y calidad de vida, hasta el estudio para la mejora de las organizaciones sanitarias y educativas (hospitales, centros de atención primaria, geriátricos, escuelas, institutos, etc.), pasando por las relaciones entre profesionales en los equipos educativos y también profesionales de la salud y de la educación física en particular.

Así, destacamos las acciones en la denominada educación «formal» (sistema educativo), en los centros educativos de primaria y secundaria, y en la denominada educación «no formal» (educación social, animación sociocultural, actividades de ocio y deporte, etc.). La investigación acción, en estos contextos de educación física, integra la idea de mejora en los educadores y en los niños y jóvenes, como sujetos activos.

La investigación-acción permite entender la labor profesional como una actitud de investigación para mejorar la práctica, analizando situaciones grupales y experiencias cotidianas, y contribuyendo así a la resolución de problemas (Fraile, 1991; Molina, 2002a). El objeto de la investigación-acción es la transformación de la práctica social, al mismo tiempo que se procura comprenderla mejor, hay una articulación permanente de la investigación, la acción y la formación a lo largo de todo el proceso (Bartolomé Pina, 1997). Resalta la actividad cotidiana como un acontecimiento extraordinario, en el marco de una orientación etnográfica (Garfinkel, 1967; Goffman, 1971; Guerrero, 1991; Molina, 2002a,b) Orientación etnográfica a través de anotaciones de campo, análisis de documentos, cuestionarios y entrevistas, modelos sociométricos y registros en video, así como la posibilidad de desarrollar un diario del proyecto que ayuda en la planificación y la reflexión constante.

En la investigación-acción es fundamental el análisis de las interrelaciones y la mejora de ellas como consecuencia de dicha investigación.

La investigación debe tomar como referencia, además de las familias y los propios educadores (entre otros), la posición y los puntos de vista de los niños y jóvenes. Por ello pueden contestar cuestionarios adaptados sobre indicadores de calidad de vida (más subjetiva), pero en un marco etnográfico, ya que la etnografía es una metodología particularmente útil para el estudio de la infancia (Gaitán, 2006: 17-20). Como ejemplo ilustrativo podemos destacar la investigación de Reverter-Masia, Molina-Luque, Farrás-Llados y Reverter (2015) con el objetivo de analizar el modo de desplazamiento al colegio de los alumnos de primaria, teniéndose en cuenta la asociación de factores ambientales (distancia y tiempo del trayecto al colegio) con el modo de desplazamiento al colegio de los niños y niñas de 6 a 12 años. Los escolares completaron un cuestionario sobre el modo de desplazamiento al colegio y aspectos ambientales. Los alumnos manifestaron percibir los caminos como seguros y una amplia mayoría vivir cerca del colegio. Esta investigación es previa a una intervención educacional con el fin de favorecer el desplazamiento activo al colegio en los niños de primaria. Y es que la inactividad física en niños y jóvenes sigue siendo una epidemia internacional, con el potencial de contribuir a las enfermedades crónicas en la edad adulta. Dado que la mayoría de los niños y los jóvenes no están cumpliendo con las directrices propias de la actividad física, en diversos países se ha prestado mucha atención a las estrategias para elevar los niveles de actividad física en niños y adolescentes. El desplazamiento activo a la escuela (es decir, caminar / andar o ir en bicicleta a la escuela) puede ser una estrategia para aumentar los niveles de actividad física en los jóvenes. Algunos estudios han demostrado una asociación entre el transporte activo en la escuela y los niveles de actividad física y, posiblemente, prevenir o atenuar los incrementos de peso corporal (Lee et al., 2008).

Podemos hablar incluso de una mixtura metodológica integrando una perspectiva estructural (cuantitativa, a través de estadísticas sobre niños y jóvenes), construccionista (etnográfica, a través de la observación participante) y relacional (cualitativa, investigación-acción, con entrevistas en profundidad, grupos de discusión, etc. a los propios niños y jóvenes, pero también con las familias, educadores, etc.).

En todo caso, y aunque los educadores juegan un papel activo evidentemente-, es esencial recoger también datos directamente de los niños y jóvenes, ya que son ellos los que pueden dar mejor información acerca de símismos:

«Hasta el momento, gran parte de la información sobre los niños se ha basado en la utilización de encuestas y cuestionarios formales y estructurados en los que en muchas ocasiones los informadores no son siquiera ellos mismos, sino adultos que se expresan, bien en su nombre, bien a partir de su propia experiencia como personas que tratan con niños. Este es el caso de los padres, madres, profesores u otros profesionales» (Gaitán, 2006: 24)

Por tanto, a través de cuestionarios, entrevistas y grupos de discu- 
sión (y también de la observación participante) podemos identificar y valorar los hábitos de vida de los niños y jóvenes que tienen una relación directa con efectos positivos sobre la salud (práctica de actividad física, estilos de vida saludables, educación de la salud, etc.). La evaluación de los distintos dominios de forma subjetiva miden aspectos de la calidad de vida, si fueran de forma objetiva, hablaríamos de medida de bienestar (Fernández-López et al. 2010: 180). De hecho, cuando nos situamos en el marco de indicadores de calidad de vida y aún estando referidos en general a toda la población, dadas las características de las distintas fuentes originales de las que provienen los datos, existen distintas limitaciones en cuanto a grupos de edad, fundamentalmente para la población de edad inferior a 16 años (vid Indicadores de Calidad de Vida INE, 2014). Con todo, precisamente la salud es el aspecto más valorado: una buena salud es la base para una buena calidad de vida (vid Cifra INE, 2012:5).

Entre las dimensiones de la calidad de vida que presenta Eurostat, las más directamente relacionas con la Educación Física y la Nueva sociología de la infancia, son las que integran las siguientes dimensiones:

La dimensión 3 (Salud) en su apartado 3.2 (Determinantes de salud) y que se concretan en el Índice de masa corporal (3.2.1) y la práctica deportiva (3.3.3).

La dimensión 4 (Educación) en su apartado 4.1 (Competencias y habilidades) y que se concretan en el abandono educativo temprano (4.1.2) y habilidades en relación con Internet (4.1.3; vid López Ordosgoitia y Rodrigues, A.M., 2014). Por eso incorporamos la Educación Física desde una perspectiva de educación «formal», pero también de educación «no formal» (educación social, animación sociocultural, acción comunitaria, etc.).

La dimensión 5(Ocio y relaciones sociales) en su apartado 5.1 (Ocio) y que se concreta en la Satisfacción con el uso del tiempo (5.1.1. relacionado con la satisfacción con el tiempo disponible) y en su apartado 5.2. (Relaciones sociales) y que se concreta en la Satisfacción con las relaciones personales (5.2.2), los sentimientos de soledad(5.2.3) y Cohesión social: confianza en los demás (5.2.6.). Los deportes de equipo y los juegos cooperativos, entre otros, son elementos que pueden propiciar todo ello y también elementos para poder dialogar en entrevistas y grupos de discusión, etc.

Dimensión 9(Satisfacción global con la vida), a través de apreciaciones subjetivas importantes en el hecho muy personal de bienestar subjetivo, etc.

\section{Conclusiones}

Las conclusiones se centran en cómo desde la Educación Física y a través de la metodología mixta (básicamente en el marco de la investigación-acción y a través de cuestionarios, entrevistas en profundidad, grupos de discusión, etnografía y observación participante), se puede analizar de manera más completa algunos de los indicadores de calidad de vida entre niños y jóvenes, en relación con ellos mismos y en planteamientos intergeneracionales (perspectiva prefigurativa, sin olvidar la postfigurativa), de presente (de los propios niños y jóvenes «aquí y ahora») y de futuro (cuestiones estructurales de base socioeducativa).

La educación física es un vehículo inestimable para la educación en y para la calidad de vida, ya que, consolida hábitos saludables. El homo ludens integra elementos de diversos ámbitos, racional, emocional, relacional, etc. En la actualidad nos encontramos ante un cambio de paradigma y éste, para interpretar el mundo global y globalizado, es cultural (Touraine, 2005). En este sentido, nos hallamos en una nueva cultura de la calidad de vida en la que el yo interior privado tiene una mayor representatividad pública, combinándose la aspiración a la autorrealización y la afirmación del ámbito comunitario. No olvidemos, por otra parte, que la educación física es una práctica de intervención que influye en las conductas de los participantes, en función de las normas educativas implícitas o explícitas (Parlebas, 2003). La educación física tiene también mucho que ver con planteamientos de tipo cooperativo o competitivo, lo cual genera también elementos relacionales de calidad de vida. Por lo tanto, tendrá que valorarse en términos educa- tivos en qué valores estamos socializando y qué hábitos estamos consolidando; si potenciamos los juegos cooperativos o el deporte únicamente competitivo; y, en todo caso, cómo lo abordemos y cómo gestionamos el conflicto. Éste puede ser enriquecedor o devastador, depende de su planteamiento y su gestión.

La práctica deportiva y la actividad de física es pertinente en todas las edades, pero es evidente que el período de la juventud es privilegiado. En este sentido, la socialización prefigurativa facilita las pautas de integración de generaciones adultas. Los propios jóvenes si tienen una exitosa integración social son un exponente de calidad de vida para la sociedad en general. El deporte puede representar un verdadero motor de relación transversal, intergeneracional, que vehicule las relaciones sociales cotidianas.

Las relaciones sociales, precisamente, se encuentran entre los principales factores de la calidad de vida, juntamente con la participación y las condiciones de salud, entre otras. Es evidente, en este sentido, que la práctica deportiva, y la actividad física en general, juegan un papel relevante en la calidad de vida de los ciudadanos. Recordemos que la calidad de vida se refiere a las condiciones de vida no materiales, sobre todo en términos de calidad de las relaciones humanas, integración social y cultural (según el Informe final del Comité de las Regiones de la Unión Europea sobre «Evaluar la calidad de vida en las regiones y ciudades europeas. Conceptualización teórica e indicadores clásicos e innovadores» (Bruselas, 1999: 14y siguientes)... Y la felicidad, en esta línea, se refiere a la percepción subjetiva de la calidad de vida. Es cierto, no obstante, que para hablar de calidad de vida global hay que tener en cuenta las condiciones de vida individuales y las colectivas, refiriéndose al análisis que debe contemplar las condiciones de vida personales y las estructuras o intereses públicos, de la ciudadanía. Las zonas recreativodeportivas y la calidad de las relaciones entre las personas en un contexto comunitario estarían dentro de los aspectos no materiales de la calidad de vida, básicamente desde una perspectiva de organización de los servicios públicos que hay que tener en cuenta (vid Molina, 2010).

En todo caso, se trata de detectar, analizar y evaluar elementos subjetivos (y también algunos más objetivos) de calidad de vida presente, a través de la Educación Física, y fortalecer hábitos saludables y estilos de vida como predictores de calidad de vida futura. En esta línea, recordamos que fundamentalmente nos basamos en la infancia y la juventud, por ella y para ella, pero entendiendo que, a su vez, se está implementando una influencia destacable (con perspectiva de cambio presente y futura) en hábitos y estilos de vida saludables de su entorno más inmediato: familia, grupos de iguales y ámbito comunitario, en general.

\section{Agradecimiento}

Este trabajo se enmarca en la ayuda a la investigación otorgada por la AGAUR al Grupo de investigación consolidado, con financiación, «GESEC» (Grupo de Estudios Sociedad, Salud, Educación y Cultura): SGR 655(2014-2016).

\section{Referencias}

Bartolomé Pina, M. (1997). Panorámica general de la investigación sobre Educación Intercultural en Europa. Revista de Investigación Educativa, 15 (1), 7-30.

Comitéde las Regiones de la Unión Europea (1999). Evaluar la calidad de vida en las regiones y ciudades europeas. Conceptualización teórica e indicadores clásicos e innovadores. Bruselas-Luxemburgo: Oficina de Publicaciones Oficiales de las Comunidades Europeas.

Dubet, R., Martuccelli, D. (1996). Théories de la socialisation et definitions sociologiques del'école. Revue française de sociologie, 37, 511-535.

Durkheim, E. (1989). Educación y sociología. Barcelona:Ed. Península. Elias, N. \& Dunning, E. (1986). Quest for excitement. Sport and Leisure in the Civilizing Process. Oxford: Basil Blackwell Publisher Ltd. 
Fernández-López, J. A., Fernández-Fidalgo, M. y Cieza, A. (2010). Los conceptos de calidad de vida, salud y bienestar analizados desde la perspective de la clasficiación internacional del funcionamiento (CIF). Revista Española de Salud Pública, 84, 169-184.

Fernández Villarino, M., González Valero, M., Toja, B. y Corrimode, F. (2017). Valoración de la escuela y la Educación Física y su relación con la práctica de la actividad física de los escolares. Retos, 31, 312315.

Fraile, A. (1991). La investigación acción: método de análisis para una nueva Educación Física. Revista Interuniversitaria de formación de profesorado, 10, 251-264.

Gaitán, L. (2006). La nueva sociología de la infancia. Aportaciones de una mirada distinta. Política y Sociedad, 43 (1), 9-26.

García Farrando, M. (1990). Aspectos sociales del deporte. Madrid: Alianza.

Garfinkel, H. (1967). Studies in Etnomethodology. New York: Prentice Hall.

Goffman, E. (1971). Relations in Public. New York: Basic Books.

Guerrero, J. F. (1991). Introducción a la investigación etnográfica en educación especial. Salamanca:Amarú.

INE (2012). Calidad de vida. Más allá del crecimiento económico. Boletín Informativo del Instituto Nacional de Estadística-Cifras INE, 1-

INE (2016). Indicadores de Calidad de Vida (en línea). http://www.ine.es/ dynt3/metadatos/es/RespuestaDatos.htm?oe=30471, acceso 1 de Febrero de 2016

Kyle, T. L.; Hernández Mendo, A.; Reigal Garrido, R. y Morales Sánchez, V. (2016). Efectos de la actividad física en el autoconcepto y la autoeficacia en preadolescentes. Retos, 29, 61-65.

Lee, MC., Orenstein, MR., Richardson, MJ. (2008). Systematic review of active commuting to school and children's physical activity and weight. J Phys Act Health, 5: 930-949.

López Ordosgoitia, R. y Rodrigues, A. M. (2014). Los niños y el Internet: la Nueva Sociología de la Infancia y el uso de métodos visuales. Revista Comunicando, 3, 220-234.

Mead, M. (1971). Cultura y compromiso. Estudio sobre la rotura generacional. Buenos Aires: Granica.

Molina, F. (2002a). Sociología de la Educación Intercultural. Buenos Aires: Lumen.

Molina, F. (2002b). Le profil sociologique des amateurs de football. Adhésions identitaires et fidélisation. STAPS (Revue Internationale des Sciences du Sport et de l'Éducation Physique), 57, 69-84.

Molina, F. (2004). Identidades y construcciones culturales en el deporte de masas. El caso del fútbol. En Álvarez Sousa, A. (coord.), Turismo, Ocio y Deporte (pp. 519-534). A Coruña: Universidade da Coruña.

Molina, F. (2007). Socialization, Glocal Identity and Sport. European Journal for Sport and Society, 4 (2).

Molina, F. (2010). Deporte, Interculturalidad y Calidad de Vida: nuevos modelos de integración social. Anduli. Revista Andaluza de Ciencias Sociales, 9, 165-173.

Molina, F. (2012). Teaching in Physical Education: Socialisation, Play and Emotions. Electronic Journal of Research in Educational Psychology, 10 (2), 755-770.

Piéron, M. y Ruiz-Juan, F. (2013). Inluencia del ámbito familiar e iguales en los hábitos físico-deportivos de los jóvenes / Influence of family environment and peers in physical activity habits of youth people. Revista Internacional de Medicina y Ciencias de la Actividad Física y el Deporte vol. 13 (51) pp. 525-549.

Http://cdeporte.rediris.es/revista/revista51/artsocializacion385.htm

Reverter-Macià, J., Molina-Luque, F., Farrás-Lladós, N. y Reverter, M. (2015). Desplazamiento activo a las escuelas. XI Congreso Internacional sobre la Enseñanza de la Educación Física y el Deporte Escolar. Chiclana de la Frontera y Campus de Puerto Real (Cádiz), 3-6/ 09/ 2015 (ISBN: 978-84-608-1278-4).

Salas, L. P. (2010). «Calidad de vida» y «la Nueva Sociología de la Infancia», perspectivas de investigación social complementarias. Hologramática, 12 (4), 83-95.

Serperl, R. (1981). Influencia de la cultura en el comportamiento. Barcelona: CEAC.

Vicens, J,(1995). El valor de la Salud. Una reflexión sociológica sobre la calidad de vida. Madrid: S. XXI.

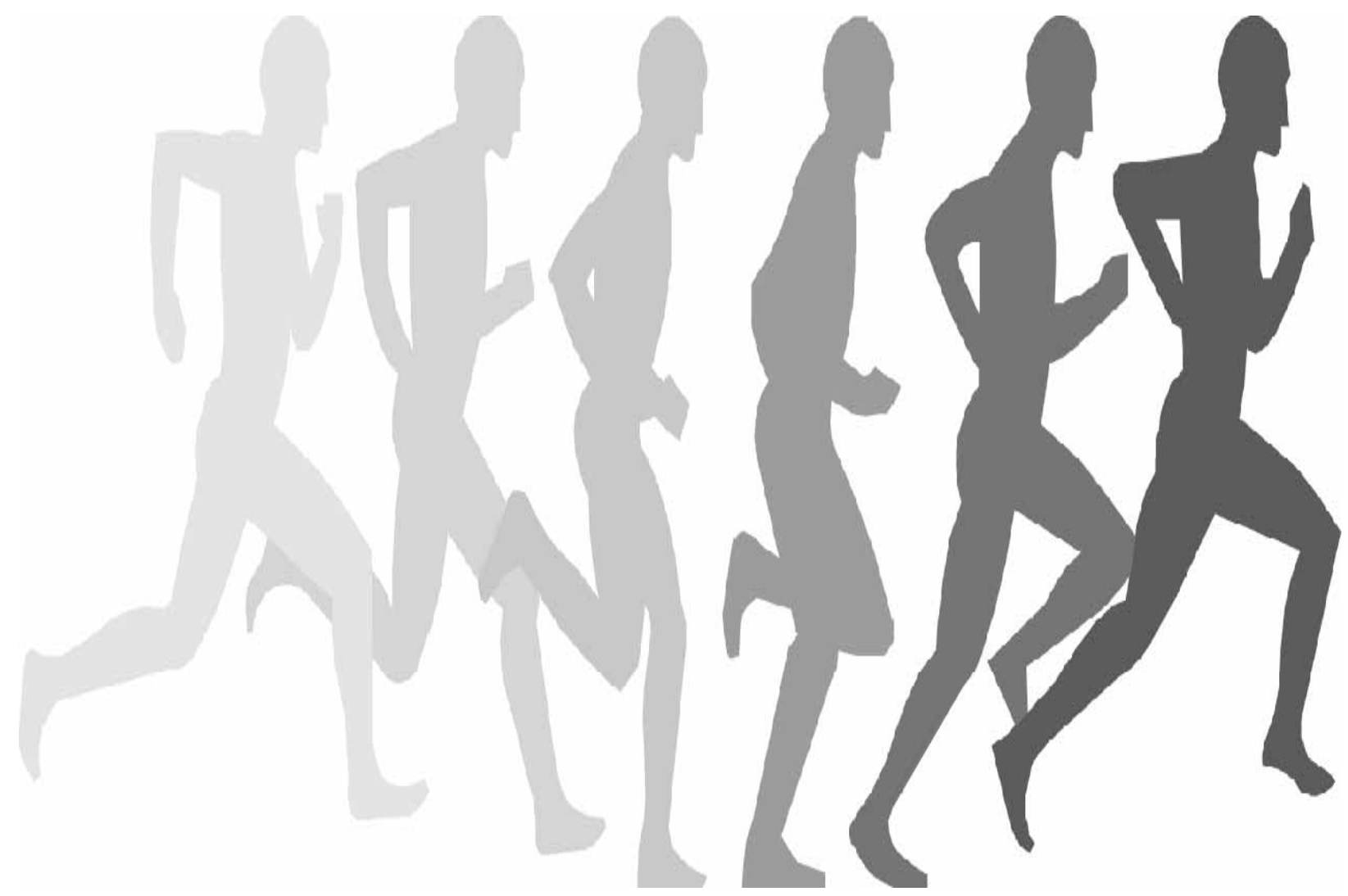

